\title{
Core-crust transition pressure for relativistic slowly rotating neutron stars
}

\author{
L. M. González-Romero and J. L. Blázquez-Salcedo \\ Depto. Física Teórica II, Facultad de Ciencias Físicas, Universidad Complutense de Madrid, \\ 28040-Madrid.
}

\begin{abstract}
We study the influence of core-crust transition pressure changes on the general dynamical properties of neutron star configurations. First we study the matching conditions in core-crust transition pressure region, where phase transitions in the equation of state causes energy density jumps. Then using a surface crust approximation, we can construct configurations where the matter is described by the equation of state of the core of the star and the core-crust transition pressure. We will consider neutron stars in the slow rotation limit, considering perturbation theory up to second order in the angular velocity so that the deformation of the star is also taken into account. The junction determines the parameters of the star such as total mass, angular and quadrupolar momentum.
\end{abstract}

Keywords: Neutron Stars, Junction Conditions, Core-crust transition pressure, Slow Rotation PACS: $04.40 . \mathrm{Dg}, 97.60 . \mathrm{Jd}, 04.25 . \mathrm{Nx}$

\section{INTRODUCTION}

The interior of the neutron stars is composed of matter in a highly compact state. Most of the theories that describe the matter composition of these stars predicts a layer structure, resulting from the different matter species that emerge as a result of the extreme densities reached [1]. Neutron stars are composed by the core and the crust, whose properties are very different. In the core of the star (around $10 \mathrm{Km}$ of radius), matter is found at densities well beyond nuclear density. The equation of state for matter in this state is not well known, and different hypothesis exist, essentially differing on which particle populations could be found and which ones dominates over the others. The outer regions of the core is composed by neutrons, protons, electrons and possibly muons while in the interior regions more exotic matter states are thought to be found (pions, hyperons, quark matter...). The crust of neutron stars is reached at nuclear densities, and a mix of neutrons, free electrons, and neutron-rich atomic nuclei is thought to give a metallic structure to this region [2].

Along the core of the star and specially in the core-crust interface, first order phase transitions are expected to be found in realistic equations of state. These phase transitions result in small discontinuities in the energy density of the star matter $[2,3]$. The appropriate way of treating these discontinuities is by analyzing the junction between every region in the interior of the star, i.e., the matching conditions in the core-crust transition region. This junction must satisfy some basic conditions so that the resulting metric is continuous and the Einstein equation is satisfied. We use the intrinsic formulation of these conditions that can be written in terms of the first and second fundamental forms $[4,5,6]$. We will make an interpretation of this matching in terms of physical

Towards New Paradigms: Proceeding of the Spanish Relativity Meeting 2011

AIP Conf. Proc. 1458, 419-422 (2012); doi: 10.1063/1.4734449

(C) 2012 American Institute of Physics 978-0-7354-1060-2/\$30.00 
quantities like the transition pressure.

\section{MATCHING THE TRANSITION PRESSURE REGION}

In nature neutron stars are found rotating in the form of pulsars. Although the angular velocity of these objects is very high (with periods from seconds to milliseconds), when compared with the gravitational energy of the compact star, the rotational energy of the star is in most cases smaller. So that for modeling a realistic neutron star we can use the slow rotation Hartle-Thorne perturbative theory for rigid rotating axi-symmetric stars. We will consider the slow rigid rotation approximation up to second order in the angular velocity, so that the deformation of the star due to its rotation is also considered. We will follow the notation of the original papers by Hartle and Thorne $[7,8]$, with the perturbed metric given by $d s^{2}=-e^{2 \psi}[1+2 h] d t^{2}+e^{2 \lambda}[1+2 m] d r^{2}+$ $r^{2}[1+2 k]\left[d \theta^{2}-\sin ^{2} \theta(d \phi-\omega d t)^{2}\right]$.

We will consider the case in which a interior region with perfect fluid with a given equation of state is being matched to an exterior perfect fluid with another equation of state. The matching will be done on a surface of constant pressure (up to second order in the angular velocity). The inner face of the surface will have in general some values $\left(p_{-}, \rho_{-}\right)$, and the exterior region $\left(p_{+}, \rho_{+}\right)$[9].

We must grant that the general solution satisfy the continuity of the first fundamental form in order to have a well defined metric on the surface. The Einstein equations tell us that the discontinuity resulting from the jump of the second fundamental form can be written as a surface stress-energy tensor in the core-crust interface. So introducing a surface energy density on the interface surface causes a discontinuity of the pressure on this surface. We will write this superficial stress-energy tensor as a perfect fluid tensor with surface density $\rho_{c}(\theta)=\varepsilon+\delta \varepsilon(\theta)=\varepsilon+\delta \varepsilon_{0}+\delta \varepsilon_{2} P_{2}(\theta)$ where $\varepsilon$ is the zero order surface density and $\delta \varepsilon_{0}, \delta \varepsilon_{2}$ are the second order spherical and quadrupolar surface densities. They are all constant on the junction interface.

Some of the matching conditions for the functions of the metric are the following:

$$
\begin{array}{r}
\Delta\left[e^{-\lambda(a)}\right]=-4 \pi a \varepsilon \\
\Delta\left[\frac{M(a)+4 \pi a^{3} p(a)}{\sqrt{1-\frac{2 M(a)}{a}}}\right]=4 \pi a^{2} \varepsilon \\
\Delta[\omega(a)]=0 \\
\Delta\left[e^{-\lambda(a)} \partial_{R} \bar{\omega}(a)\right]=\frac{16 \pi \varepsilon}{a}\left(\Omega_{c}-\omega(a)\right)
\end{array}
$$

Where $\Delta[f(a)]=f_{+}-f_{-}$. These equations provides the matching for the zero and first order functions. Essentially the introduction of a discontinuity in density and pressure inside of the star implies the apparition of a surface energy density that also modifies the mass. The inertial dragging must be continuous across the surface, while the derivative of the inertial dragging presents a discontinuity given by the angular velocity of the surface density (which modifies the total angular momentum of the star). If the angular 
velocity of the two regions is the same, then the surface density must rotate with the same angular velocity.

Studying the matching up two second order reveals matching conditions for the remaining metric functions, providing the jump in the mass perturbation, quadrupolar moment and second order terms of the surface energy density. These equations will be presented elsewhere.

\section{SURFACE CRUST APPROXIMATION}

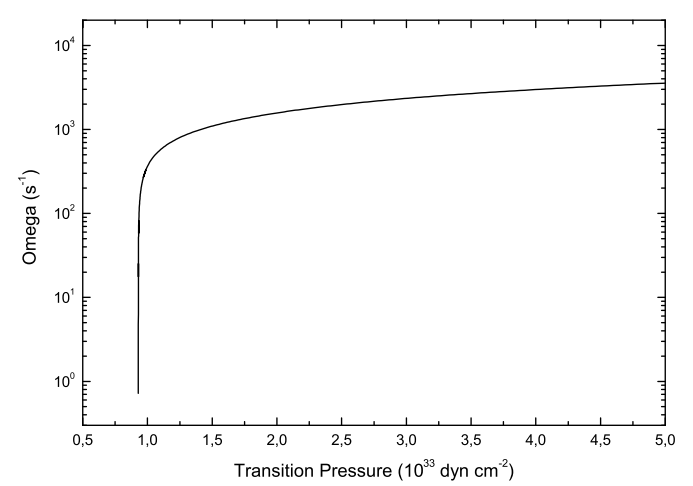

FIGURE 1. Angular velocity vs transition pressure for central density $1.279 \cdot 10^{15} \mathrm{gcm}^{-3}$ and $1.44 M_{\odot}$

In general we will need to integrate, inside the star, the metric functions in different regions separated by the different phase transitions. If we just consider the phase transition at the core-crust interface, we will need to integrate in two regions, inside the core of the star and along the crust. We will need to impose the previous boundary conditions that grant the appropriate continuity of the metric across the regions. In order to simplify the situation so that only one region needs to be integrated, we can construct a simplified model of crust using the obtained matching conditions:

It can be proved that in the crust the density falls down to zero very quickly, and every contribution from the crust to integral properties of the star like the mass or the angular momentum can be neglected. The outer crust extends only over a region of some hundreds of meters with the density rapidly falling to zero. So approximating the crust of the star to a surface density enveloping the core of the neutron star is a well justified model of crust where the obtained radius can be interpreted as an effective radius of integration for the star. It can be seen that this approximation can be achieved in the following way.

We can integrate the core of the star with an appropriate equation of state, and match directly to the exterior vacuum solution. Then the exterior pressure and density becomes zero in the expressions for the matching, and the outer mass function becomes the mass outside the star. The interior pressure can be taken at the inner crust. The surface density obtained from the matching conditions can be interpreted as the approximated crust: the crust becomes a surface energy density enveloping the core of the star.

This simplified crust model can tell us a lot of information about the dependence of the properties of the star with the core-crust transition pressure. In Figure 1 we present 
the relation of the angular velocity of the neutron star with the transition pressure. These results are for configurations with total second order mass $1.44 M_{\odot}$ and central density $1.279 \cdot 10^{15} \mathrm{~g} \cdot \mathrm{cm}^{-3}$. The equation of state used for these results is the Glendenning 240 for high densities together with the BPS equation of state for lower densities [1].

In the figure it can be seen two different regions, one where the angular velocity is quite sensitive to core-crust transition pressure variations, and another where it is less important. Around core-crust transition pressure other properties of the star like the angular momentum and the quadrupolar momentum are also heavily modified with small perturbations of the core-crust transition pressure variation. The specific value for which this variations can affect the global properties of the star is different for other central densities and equations of state. In this case, small variations of the order of $10^{23} \mathrm{dyn} \cdot \mathrm{cm}^{-2}$ causes relative variations order $10^{-6}$ in the period, angular momentum and eccentricity.

The configurations have been generated integrating the differential equations for using a Fortran code based in the Colsys routine [10]. The equation of state has been interpolated numerically from the tables. The interpolation is based on a natural spline that makes the adiabatic index continuous (necessary to obtain a good precision in the second order perturbation functions).

Colsys is the appropriated way of resolving the problem, because Colsys allows to resolve differential equations with quite general boundary conditions. We impose the usual regularity conditions together with the boundary conditions at the border of the star that grants the solution outside the star to be asymptotically flat with the matching previously explained. Also Colsys allows to introduce fixed points of the mess where certain junction conditions could be imposed. This feature will be used in future works to integrate multiple regions with different equations of state.

The angular velocity of the star is quite dependent of the transition pressure, specially in the region nearby the core-crust transition. This means that small changes in the corecrust transition pressure could give rise to important changes in the dynamical properties of the star like angular momentum and quadrupolar moment. Energy depositions on the outer layers of the star could affect the phase transition, causing variations in the angular velocity of the star. These processes could explain pulsar glitches.

\section{REFERENCES}

1. N. Glendenning, Compact stars: nuclear physics, particle physics, and general relativity, Astronomy and astrophysics library, Springer, 2000, ISBN 9780387989778.

2. P. Haensel, P. Haensel, A. Potekhin, and D. Yakovlev, Neutron stars: Equation of state and structure, Astrophysics and space science library, Springer, 2007, ISBN 9780387335438.

3. H. Heiselberg, and M. Hjorth-Jensen, Physics Reports 328, 237 (2000).

4. G. Darmois, Les équations de la gravitation einsteinienne, Mémorial des sciences mathématiques 25, Gauthier-Villars et cie, 1927.

5. A. Lichnerowicz, Théories relativistes de la gravitation et de l'électromagnétisme:, Collection d'ouvrages de mathématiques à l'usage des physiciens, Paris, 1955.

6. C. Misner, K. Thorne, and J. Wheeler, Gravitation, W. H. Freeman, 1973, ISBN 9780716703440.

7. J. B. Hartle, Astrophys. J. 150, 1005 (1967).

8. J. B. Hartle, and K. S. Thorne, Astrophys. J. 153, 807 (1968).

9. L. M. González-Romero, Phys. Rev. D 67, 064011 (2003).

10. U. Ascher, J. Christiansen, and R. D. Russell, ACM Trans. Math. Softw. 7, 209-222 (1981). 
Copyright of AIP Conference Proceedings is the property of American Institute of Physics and its content may not be copied or emailed to multiple sites or posted to a listserv without the copyright holder's express written permission. However, users may print, download, or email articles for individual use. 